\title{
Performance Analysis of Maximal Ratio Combining over Shadowed-Rice Fading Channels
}

\author{
George A. Ropokis ${ }^{\dagger \ddagger}$, Athanasios A. Rontogiannis ${ }^{\dagger}$ Kostas Berberidis $^{\ddagger}$ and P. Takis Mathiopoulos ${ }^{\dagger}$ \\ ${ }^{\dagger}$ Institute for Space Applications and Remote Sensing, National Observatory of Athens, 15236, Athens, Greece \\ ${ }_{\ddagger}$ Dept. of Computer Engineering \& Informatics, University of Patras 26500, Rio-Patras, Greece \\ Email: \{ropokis,tronto\}@space.noa.gr, berberid@ceid.upatras.gr, mathio@space.noa.gr
}

\begin{abstract}
An accurate model for analyzing the performance of wireless land mobile satellite (LMS) communication systems is the shadowed-Rice (SR) fading model. In this paper, new simple expressions for the probability density function (PDF) and the cumulative distribution function (CDF) of the sum of possibly correlated non identically distributed squared SR random variables are derived. To this end, a novel approach is introduced for Laplace inverting the moment generating function (MGF) of the sum. Based on this approach the PDF is expressed in a simple infinite chi-squared series form, from which the CDF is also easily obtained. The derived statistics are used to analyze the performance of maximal ratio combining (MRC) over SR fading channels and novel closed-form expressions for various performance criteria such as the outage probability, the ergodic capacity and the bit error probability (BEP) are developed. The results of extensive Monte Carlo simulations are presented, which corroborate our theoretical analysis.
\end{abstract}

\section{INTRODUCTION}

Diversity combining techniques such as orthogonal spacetime block coding (OSTBC) and maximal ratio combining (MRC) have gained considerable attention recently, due to their ability to combat fading in wireless communication systems. When OSTBC or MRC is employed the signal to noise ratio (SNR) of the system is expressed as the sum of squared random variables (RVs). Hence to analyze the performance of such systems the probability density function (PDF) and cumulative distribution function (CDF) of the sum of squared RVs must be obtained in the simplest possible form, so as to facilitate the calculation of several performance metrics.

A simple representation for the PDF of the sum of squared RVs is based on infinite series expansions of chi-squared or Gamma PDFs and has been widely used both in statistics and in the analysis of wireless communications systems. More specifically, in [1],[2] and [3] the distribution of the sum of squared Gaussian RVs is studied and several series expansions for the PDF are proposed. Using a Gamma series representation the PDF and CDF of the sum of independent Gamma, i.e., squared Nakagami RVs are derived in [4] and [5]. Based on these expressions the performance of MRC over

This paper is part of the 03ED838 research project, implemented within the framework of the Reinforcement Programme of Human Research Manpower (PENED) and co-financed by National and Community Funds (75\% from E.U.-European Social Fund and 25\% from the Greek Ministry of Development-General Secretariat of Research and Technology). G. A. Ropokis was funded in part by the Satellite Network of Excellence (SatNEx) project, a Network of Excellence (NoE) funded by the the European Commitee (EC) under FP6 program.
Nakagami fading channels is studied in [6]. In [7] the chisquared series representation is adopted for the performance analysis of OSTBC over Hoyt fading channels, while in [8] the same series expression is applied for computing matched filter bounds for BPSK over multipath Rician fading channels.

Yet another fading model that has attracted increasing interest lately is the so-called shadowed-Rice (SR) model [9]. One such mathematically versatile model has been recently proposed in [10]. It has been shown in [10] that this model describes very accurately the land mobile satellite (LMS) channel, which is expected to play a prominent role in future third and fourth generation communication systems. Nevertheless, to the best of our knowledge very few results related to the SR distribution exist in the open technical literature. Only recently in [11], an analysis for the sum of squared SR RVs has been presented and the outage probability and capacity of MRC over SR fading channels have been studied. However, the closed-form expression for the PDF proposed in [11] is extremely complex, thus leading to expressions for the various performance metrics, which are quite complicated and difficult to use in practice.

Motivated by the above, in this paper, new simple expressions for the PDF and the CDF of the sum of squared SR RVs are introduced, following a novel analytical approach. Our approach is based on a proper manipulation of the Laplace transform of the PDF, which was first used in [1] and [2] for the case of squared Gaussian RVs. The PDF is expressed in a simple chi-squared series form, which is proven to converge uniformly. Then, the resulting PDF and CDF formulas are used to analyze the performance of MRC over SR fading channels in terms of the outage probability, the ergodic capacity and the bit error probability (BEP). It should be emphasized that not only the expressions for the PDF, CDF and the various performance criteria are much simpler than those in [11], but also the fading model considered in this work is more general too. Moreover, the proposed analytical method can be generalized to other practical fading distributions, an issue, which due to space limitations will not be elaborated any further.

The outline of the paper is as follows. In Section II the distribution of the sum of squared SR RVs is analyzed and closed-form expressions for the PDF and CDF are provided. New simple expressions of various performance metrics for MRC over SR fading channels are described in Section III. Simulation results are presented in Section IV and concluding 
remarks in Section V.

\section{DISTRIBUTION OF THE SUM OF SQUARED SR RVS}

The SR fading model can be defined as a Rice fading model, whose line-of-sight (LOS) component is random. In this work we adopt the SR model proposed in [10], in which the amplitude of the LOS component is Nakagami distributed. More specifically, the complex baseband representation of this SR fading channel model is given as follows [10]

$$
a_{F}=a_{R} \exp (j \phi)+a_{N} \exp (j \zeta)
$$

where $a_{R}$ is a Rayleigh RV with average power $2 b, \phi$ is uniformly distributed in $[0,2 \pi), a_{N}$ is Nakagami distributed with parameters $\Omega$ and $m$, and $\zeta$ is the nonrandom phase of the LOS component of the channel. Then, the power $r=\left|a_{F}\right|^{2}$ of the fading process follows a squared SR distribution, whose $\mathrm{PDF}$ is given by [10]

$$
\begin{aligned}
p_{r}(r)= & \left(\frac{2 b m}{2 b m+\Omega}\right)^{m} \frac{1}{2 b} \exp \left(-\frac{r}{2 b}\right) \\
& \times{ }_{1} F_{1}\left(m, 1 ; \frac{\Omega r}{2 b(2 b m+\Omega)}\right)
\end{aligned}
$$

where ${ }_{1} F_{1}(\cdot, \cdot ; \cdot)$ is the confluent hypergeometric function [12]. It can then be shown that the moment generating function (MGF) of $r$, is expressed as [10]

$$
M_{r}(s)=\frac{(1-2 b s)^{m-1}}{\left(1-\left(2 b+\frac{\Omega}{m}\right) s\right)^{m}} .
$$

Using this form of the MGF, the PDF and CDF of the sum of independent and correlated squared SR RVs will be derived in the following sections.

\section{A. Sum of independent squared SR RVs}

Let us first define the following RV

$$
z=\sum_{i=1}^{n} r_{i}
$$

where $r_{i}, i=1,2, \ldots, n$ are independent non identically distributed squared SR RVs with parameters $\left\{\Omega_{i}, b_{i}, m_{i}\right\}$. Then by utilizing the relation between the MGF of a RV and the Laplace transform of its PDF [9], the Laplace transform of the PDF of $z$ is directly written from (3) and (4) as

$$
L_{z}(s)=\prod_{i=1}^{n} \frac{\left(1+2 b_{i} s\right)^{m_{i}-1}}{\left(1+\left(2 b_{i}+\frac{\Omega_{i}}{m_{i}}\right) s\right)^{m_{i}}} .
$$

Apparently, the PDF of $z$ can be obtained by calculating the inverse Laplace transform of $L_{z}(s)$. Here, we follow a method similar to that originally used in [1] for the sum of squared Gaussian RVs. We show that this method is easily extended for the case of squared SR RVs. Indeed, let us define the function

$$
\theta(s)=\frac{1}{1+s \beta}
$$

where $\beta$ is an arbitrary positive parameter. Notice that for any $a>0$ the following identity holds

$1+a s=1+s \beta \frac{a}{\beta}+\frac{a}{\beta}-\frac{a}{\beta}=\frac{a}{\beta \theta(s)}\left(1-\left(1-\frac{\beta}{a}\right) \theta(s)\right)$.

From (5) and (7), $L_{z}(s)$ is readily expressed as

$$
L_{z}(s)=A \theta^{n}(s) \prod_{i=1}^{n} \frac{\left(1-\gamma_{i} \theta(s)\right)^{m_{i}-1}}{\left(1-\delta_{i} \theta(s)\right)^{m_{i}}}
$$

where

$$
A=\beta^{n} \prod_{i=1}^{n} \frac{\left(2 b_{i}\right)^{m_{i}-1}}{\left(2 b_{i}+\frac{\Omega_{i}}{m_{i}}\right)^{m_{i}}}
$$

and

$$
\gamma_{i}=1-\frac{\beta}{2 b_{i}}, \quad \delta_{i}=1-\frac{\beta}{2 b_{i}+\frac{\Omega_{i}}{m_{i}}} .
$$

The basic idea is to express $L_{z}(s)$ given in (8) as a series expansion so that, through Laplace inversion to derive a simple formula for the PDF. To this end, let us first introduce the function

$$
L(\theta)=\prod_{i=1}^{n} \frac{\left(1-\gamma_{i} \theta\right)^{m_{i}-1}}{\left(1-\delta_{i} \theta\right)^{m_{i}}}
$$

whose logarithm is written as

$$
\ln L(\theta)=\sum_{i=1}^{n}\left(m_{i}-1\right) \ln \left(1-\gamma_{i} \theta\right)-\sum_{i=1}^{n} m_{i} \ln \left(1-\delta_{i} \theta\right) \text {. }
$$

For $\theta$ satisfying $\theta<1 / \max \left\{\max _{i}\left\{\left|\gamma_{i}\right|\right\}, \max _{i}\{|\delta i|\}\right\}$, a series expansion for the logarithm of ${ }^{i}(\theta)$ exists and is given by

$$
\ln L(\theta)=\sum_{j=1}^{\infty} d_{j} \frac{\theta^{j}}{j}
$$

where

$$
d_{j}=\sum_{i=1}^{n} m_{i} \delta_{i}^{j}-\sum_{i=1}^{n}\left(m_{i}-1\right) \gamma_{i}^{j}
$$

Then according to [13, pp. 93], (11) can be rewritten as

$$
L(\theta)=\sum_{i=0}^{\infty} c_{i} \theta^{i}
$$

where the coefficients of the series are computed according to the following recursive formula

$$
c_{0}=L(0) \quad \text { and } \quad c_{i}=\frac{1}{i} \sum_{l=0}^{i-1} d_{i-l} c_{l} \text { for } i>0 .
$$

From (8) and (15), $L_{z}(s)$ is expressed in a series expansion form, i.e.,

$$
L_{z}(s)=A \sum_{i=0}^{\infty} c_{i} \theta^{n+i}(s) .
$$

Recall that for integer $v$

$$
\mathcal{L}^{-1}\left\{\theta^{v}(s)\right\}=\frac{z^{v-1} \exp (-z / \beta)}{\beta^{v}(v-1) !}
$$


where $\mathcal{L}^{-1}$ stands for the inverse Laplace transform. Thus, from (17) and (18) the PDF of $z$ is finally given by the following closed-form expression

$$
p_{z}(z)=A \sum_{i=0}^{\infty} c_{i} \frac{z^{n+i-1} \exp (-z / \beta)}{\beta^{n+i}(n+i-1) !}
$$

where $A$ is given by (9) and $c_{i}$ are recursively computed from (16). Observe that (19) has a very simple form avoiding the use of complicated functions such as the confluent hypergeometric function, as is the case with the expression of the PDF proposed in [11]. Moreover, the analysis has been made for a more general model than that adopted in [11], where it is assumed that $b_{1}=b_{2}=\ldots=b_{n}$.

Applying term by term integration in (19), the CDF of the sum of squared SR RVs is expressed as follows

$$
P_{z}(z)=A \sum_{i=0}^{\infty} c_{i} \frac{\gamma(n+i, z / \beta)}{(n+i-1) !}
$$

where $\gamma(\cdot, \cdot)$ is the lower incomplete Gamma function [12, pp. 260]. Notice, that since $n+i$ is an integer, $\gamma(n+i, z / \beta)$ can be written as a linear combination of elementary functions leading to an extremely simple form for the CDF. To be able to interchange integration and summation, so as to arrive in (20), uniform convergence of the series in (19) is required. This result is established in the Appendix, where also the range of admissible values for parameter $\beta$ is given. It should be noted that $\beta$ controls the convergence of the series, and thus specifies the number of series terms that should be retained for a prescribed accuracy.

\section{B. Correlated SR RVs}

The previous analysis can easily be extended for correlated SR RVs obeying the correlated fading scenario employed in [11]. Let us consider the following RV

$$
z_{C}=\sum_{i=1}^{n} r_{i}
$$

where $r_{i}=\left|a_{R_{i}} \exp \left(j \phi_{i}\right)+a_{N_{i}} \exp \left(j \zeta_{i}\right)\right|^{2}, i=1,2 \ldots, n$ are squared SR RVs with parameter set $\left\{\Omega_{i}, b, m\right\}$. Under the above mentioned scenario the Rayleigh RVs $a_{R_{i}}$ are independent, while the RVs $a_{N_{i}}^{2}, i=1, \ldots, n$ are correlated. Then, by defining the matrix $\mathbf{C}$ whose $(i, j)$-th element is the square root of the correlation coefficient of the RVs $r_{i}$ and $r_{j}$ and the matrix $\mathbf{D}=\operatorname{diag}\left\{\frac{\Omega_{1}}{m}, \ldots, \frac{\Omega_{n}}{m}\right\}$, it can be shown that $z_{C}$ is equal in distribution with the following $\mathrm{RV}$

$$
z_{I}=\sum_{i=1}^{n} r_{i}^{\prime}
$$

where $r_{i}^{\prime}$ are independent squared SR RVs with parameters $\left\{\lambda_{i} m, b, m\right\}$ with $\lambda_{i}$ being the $i$ th eigenvalue of the matrix DC. Thus, instead of analyzing the initial RV $z_{C}$, an equivalent analysis can made for $z_{I}$, as described above.

\section{MRC OVER SR FADING}

As it is known, in a communications system employing $n$ receive antennae and an MRC combiner, the $\mathrm{SNR} z$ at the receiving end can be expressed as

$$
z=\sum_{i=1}^{n} z_{t}\left|h_{i}\right|^{2}
$$

where $z_{t}$ is the SNR at the transmitter side, i.e., the ratio of the average transmitted power over the noise power, and $h_{i}$ the fading coefficient of the $i$-th branch. Hence, assuming that $\left|h_{i}\right|, i=1, \ldots n$ are independent SR distributed RVs, it is easy to see that

$$
z \stackrel{d}{=} \sum_{i=1}^{n} r_{i}
$$

where $\stackrel{d}{=}$ denotes equality with respect to distribution, and $r_{i}=$ $z_{t}\left|h_{i}\right|^{2}$. By denoting with $\left\{\Omega_{\left|h_{i}\right|}, b_{\left|h_{i}\right|}, m_{\left|h_{i}\right|}\right\}$ the parameters of $\left|h_{i}\right|$ and with $\left\{\Omega_{i}, b_{i}, m_{i}\right\}$ the parameters of $r_{i}$ it is easy to show that

$$
\left\{\Omega_{i}, b_{i}, m_{i}\right\}=\left\{z_{t} \Omega_{\left|h_{i}\right|}, z_{t} b_{\left|h_{i}\right|}, m_{\left|h_{i}\right|}\right\} .
$$

Thus the PDF and CDF of $z$ in (24) will be given by (19) and (20) respectively with the coefficients $c_{i}$ calculated as in (16). Based on the expressions of the PDF and CDF already derived, in the following an analysis of MRC over SR fading channels in terms of outage probability, ergodic capacity and $\mathrm{BEP}$ is presented.

\section{A. Outage Probability}

The outage probability $P_{\text {out }}\left(z_{0}\right)$ of a communication system is defined as the probability that the SNR at the receiving end drops under a predefined threshold $z_{0}$. Hence, for a maximal ratio combiner operating over $\mathrm{SR}$ fading, $P_{\text {out }}\left(z_{0}\right)$ can be calculated from (20), i.e.,

$$
P_{\text {out }}\left(z_{0}\right)=A \sum_{i=0}^{\infty} c_{i} \frac{\gamma\left(n+i, z_{0} / \beta\right)}{(n+i-1) !} .
$$

It should be noticed that (26) is much simpler and easier to calculate than the expression of the outage probability proposed in [11], which involves a double infinite series expansion.

\section{B. Ergodic Capacity}

By definition, the capacity of an MRC system, expressed in $b i t s / s e c / H z$ is given by

$$
C=\log _{2}(1+z)
$$

with $z$ defined as in (23). Moreover, the ergodic capacity is defined as the average value of the capacity with respect to the SNR. Hence, from (19) in the case of SR fading the ergodic capacity is expressed as

$$
\langle C\rangle=A \log _{2}(e) \sum_{i=0}^{\infty} \frac{c_{i}}{\beta^{n+i}(n+i-1) !} \mathcal{I}_{n+i}
$$


with $\mathcal{I}_{v}$ defined as

$$
\mathcal{I}_{v}=\int_{0}^{\infty} z^{v-1} \exp (-z / \beta) \ln (1+z) d z .
$$

Since $v$ is integer, $\mathcal{I}_{v}$ takes the following closed form [14]

$$
\mathcal{I}_{v}=(v-1) ! \exp (1 / \beta) \sum_{k=1}^{v} \beta^{k} \Gamma(-v+k, 1 / \beta)
$$

where $\Gamma(\cdot, \cdot)$ is the upper incomplete Gamma function [12, pp. 260]. Again, by comparing (28) with [11, eq. (16)], the advantage of (28) in terms of simplicity is clear.

\section{Bit Error Probability}

In general, to calculate the BEP of a diversity scheme, averaging of the BEP for the additive white Gaussian (AWGN) channel with respect to the distribution of the instantaneous SNR per bit at the receiver side, is required. Following this procedure and assuming BPSK or Gray-coded QPSK, the bit error probability takes the form ${ }^{1}$

$$
P_{e}=A \sum_{i=0}^{\infty} c_{i} \mathcal{J}_{n+i}
$$

where $c_{i}$ 's are the coefficients of the series in (19) that result by setting $z_{t}=E_{b} / N_{0}$ in (23). $E_{b}$ is the transmitted energy per bit and $N_{0}$ the noise density at each branch of the receiver. The integral $\mathcal{J}_{v}$ is defined as

$$
\mathcal{J}_{v}=\int_{0}^{\infty} \frac{z^{v-1} \exp (-z / \beta)}{\beta^{v}(v-1) !} Q(\sqrt{2 z}) d z
$$

where $Q(\cdot)$ denotes the Gaussian Q-function. For $v$ integer this integral is expressed in closed-form as [9, pp. 149-150]

$$
\mathcal{J}_{v}=\frac{1}{2}\left[1-\mu \sum_{k=0}^{v-1}\left(\begin{array}{c}
2 k \\
k
\end{array}\right)\left(\frac{1-\mu^{2}}{4}\right)^{k}\right]
$$

where

$$
\mu=\sqrt{\frac{\beta}{1+\beta}}
$$

Notice that similar results can be obtained for the performance of MRC over correlated SR fading according to the analysis of section II.B. Additionally, due to the equivalence of OSTBC with MRC, similar expressions for the outage probability, ergodic capacity and BEP also hold for OSTBC over SR fading [7].

\section{Performance eValuation}

In this section, extensive Monte Carlo computer simulated results are used to verify the presented theoretical analysis under various fading conditions. Two different fading schemes are considered for maximal ratio combiners employing up to three receive antennae. In Scheme 1 the values of the channel parameters are selected from the sets $\left(\Omega_{1}, \Omega_{2}, \Omega_{3}\right)=$ $(0.278,0.27,0.3), \quad\left(m_{1}, m_{2}, m_{3}\right)=(5.21,5.2,5.25)$ and

\footnotetext{
${ }^{1}$ As shown in [15], in case that Gray coding is employed, the BEP of $M$ QAM coincides with the BEP of $\sqrt{M}$-PAM.
}

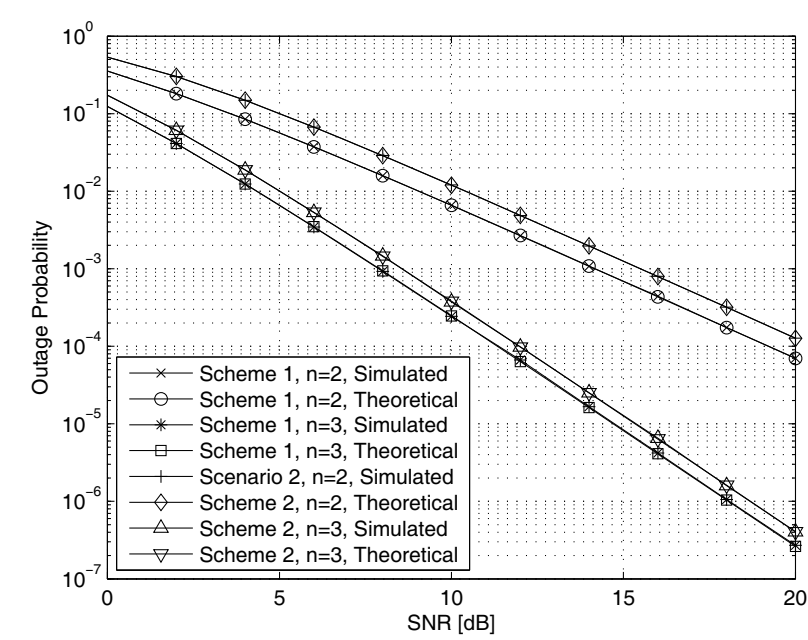

Fig. 1. Outage Probability as a function of the average transmitted SNR for the two fading schemes $\left(z_{0}=1\right)$.

$\left(b_{1}, b_{2}, b_{3}\right)=(0.251,0.251,0.251)$, as in [11]. In Scheme 2 , the values of the channel parameters are taken from the sets $\left(\Omega_{1}, \Omega_{2}, \Omega_{3}\right)=(0.2,0.3,0.4),\left(m_{1}, m_{2}, m_{3}\right)=$ $(5.21,3.2,1.5)$ and $\left(b_{1}, b_{2}, b_{3}\right)=(0.1,0.2,0.3)$. Note that the parameters of Scheme 2 have a larger spread and also $b_{i} \neq b_{j}$ for $i \neq j$. For both fading schemes, $n=2$ and $n=3$ receive antennae are examined with the parameters of the $i$-th fading channel selected as $\left\{\Omega_{i}, b_{i}, m_{i}\right\}$. For all the theoretical results concerning Scheme 1, the parameter $\beta$ was taken equal to 0.5 and a number of 11 terms have been retained from the respective series expansions. For Scheme 2 a parameter $\beta$ equal to 0.39 ensures a relatively fast convergence of the series, which have been truncated at the 15-th term.

In Fig. 1 the outage probability for the two schemes and $z_{0}=1$ is plotted as a function of the transmit $\operatorname{SNR} z_{t}$, along with Monte Carlo simulation results. It can be seen that for both schemes the theoretical and simulations results almost coincide thus verifying the validity of the derived expressions. Additionally, in Fig. 2, the ergodic capacity versus the transmit SNR for both fading schemes is plotted as calculated using (28) along with simulation results. Again, the close match of theoretical and simulations results can be verified. Finally, the agreement between theoretical and simulations results can also be observed in Fig. 3 where the BEP of BPSK for the two fading schemes is plotted.

\section{CONCLUSION}

By applying an effective method to manipulate the Laplace transform of the PDF, new expressions for the PDF and CDF of the sum of squared SR RVs have been derived. Without setting any restriction to the parameters of the model, the proposed expressions are much simpler compared to already known ones. Based on these results, mathematically tractable closed-form expressions for the outage probability, the ergodic capacity and the BEP of MRC over SR fading have been presented. The validity of our theoretical analysis has been verified through extensive Monte Carlo Simulations. 


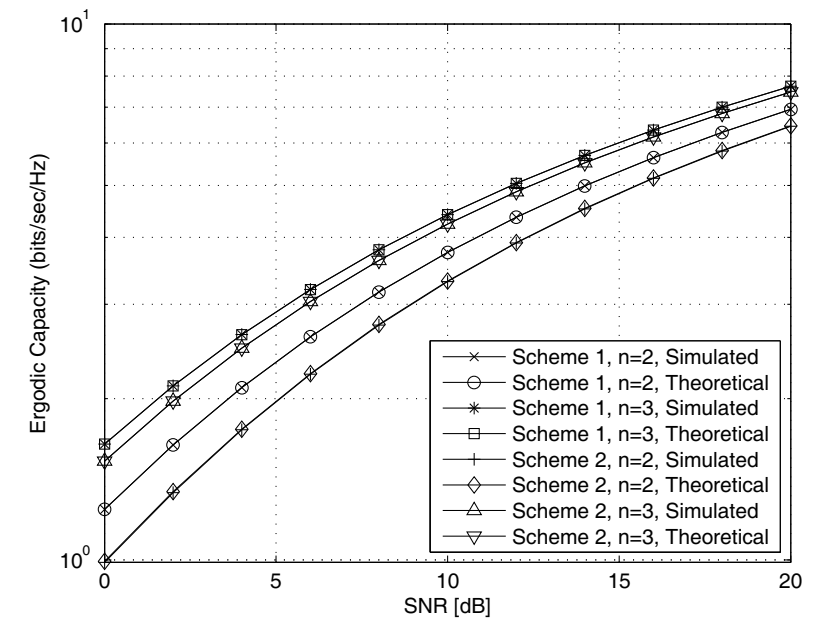

Fig. 2. Ergodic capacity as a function of the average transmitted SNR for the two different fading schemes.

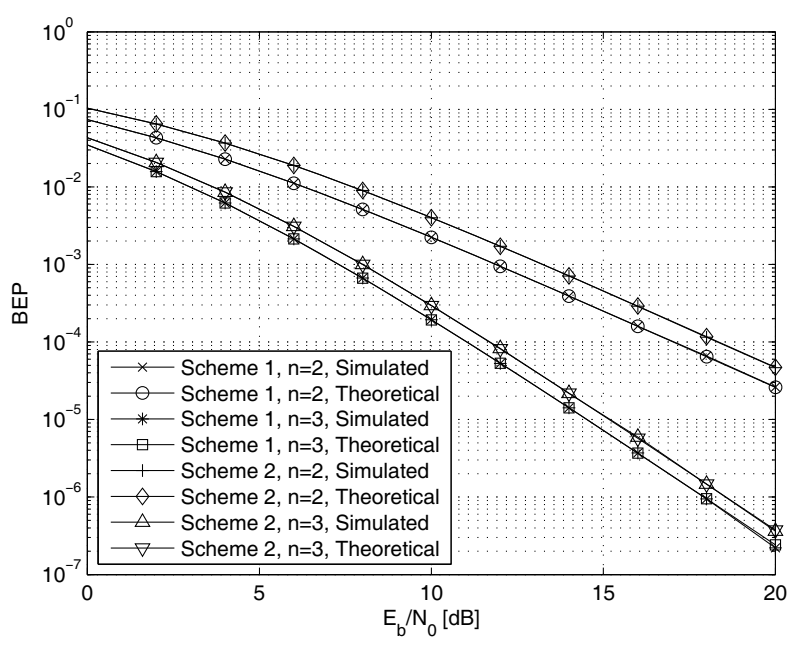

Fig. 3. BEP for BPSK for the two fading schemes.

\section{APPENDIX}

The uniform convergence of the series in (19) can be proven by properly bounding the series truncation error. More specifically, since $c_{i}$ 's are the coefficients of the power series expansion of $L(\theta)$ the following holds

$$
c_{i}=\frac{L^{(i)}(0)}{i !}
$$

where $L^{(i)}(\cdot)$ is the $i$-th derivative of $L(\cdot)$. By employing Cauchy's inequality the absolute value $c_{i}$ can be bounded as [16]

$$
\left|c_{i}\right| \leq \frac{L_{0}(u)}{u^{i}}
$$

where $u$ is any positive value satisfying $u<$ $1 / \max \left\{\max _{i}\left\{\left|\gamma_{i}\right|\right\}, \max _{i}\{|\delta i|\}\right\}$ and

$$
L_{0}(u)=\max _{|\theta|=u}|L(\theta)| .
$$

By employing (36) a bound on the absolute value of the truncation error $\varepsilon(z)$ of (19) can be obtained as

$$
\varepsilon(z) \leq A \frac{L_{0}(u) u^{n-1} \exp \left(-\frac{z}{\beta}\right)}{\beta} \sum_{i=N+1}^{\infty} \frac{(z / \beta u)^{n+i-1}}{(n+i-1) !} .
$$

After some algebraic manipulations, (38) is rewritten as

$$
\begin{aligned}
\varepsilon(z) \leq & A \frac{L_{0}(u) u^{n-1} \exp \left(-\frac{z}{\beta}\right)}{\beta} \\
& \times\left(\exp \left(\frac{z}{\beta u}\right)-\sum_{i=0}^{n+N-1} \frac{(z / \beta u)^{i}}{i !}\right) .
\end{aligned}
$$

By inspecting (39), it can be seen that uniform convergence of $p_{z}(z), \forall r \geq 0$ is achieved as long as $u>1$, or equivalently as long as $0<\beta<4 \min _{i}\left\{b_{i}\right\}$.

\section{REFERENCES}

[1] S. Kotz, N. L. Johnson, and D. W. Boyd, "Series representations of distributions of quadratic forms in normal variables: I. Central case," The Annals of Mathematical Statistics, vol. 38, no. 3, pp. 823-837, June 1967.

[2] - "Series representations of distributions of quadratic forms in normal variables: II. Non-Central case," The Annals of Mathematical Statistics, vol. 38, no. 3, pp. 823-837, June 1967.

[3] H. Ruben, "Probability content of regions under spherical normal distributions, IV: The distribution of homogeneous and non-homogeneous quadratic functions of normal variables," The Annals of Mathematical Statistics, vol. 33, no. 2, pp. 542-570, June 1962.

[4] P. G. Moschopoulos, "The distribution of the sum of independent Gamma random variables,", Ann. Inst. Statist. Math. (Part A),, vol. 37, pp. 541544, 1985.

[5] S. B. Provost, "On sums of independent Gamma random variables,", Statistics, vol. 20, no. 4, pp. 583-591, 1989.

[6] M. S. Alouini, A. Abdi, and M. Kaveh, "Sum of Gamma variates and performance of wireless communication systems over Nakagami-fading channels," vol. 50, no. 6, pp. 1471-1480, N0v. 2001.

[7] G. A. Ropokis, A. A. Rontogiannis, and P. T. Mathiopoulos, "Performance analysis of orthogonal space time block coding over Hoyt fading channels," in Proc. IEEE Globecom, Nov. 2007, pp. 3416-3420.

[8] K. Yip and T. Ng, "Matched filter bound for multipath Rician fading channels," IEEE Trans. Commun., vol. 46, no. 4, pp. 441-445, Apr. 1998.

[9] M. K. Simon and M.-S. Alouini, Digital Communication over Fading Channels. John Wiley and Sons, 2005.

[10] A. Abdi, W. C. Lau, M. S. Alouini, and M. Kaveh, "A new simple model for land-mobile satellite channels: First- and second-order statistics,," IEEE Trans. Wireless Commun., vol. 2, no. 3, pp. 519-528, May 2003.

[11] G. Alfano and A. D. Maio, "Sum of squared shadowed-Rice random variables and its application to communication systems performance prediction,", IEEE Trans. Wireless Commun., vol. 6, no. 10, pp. 35403545, Oct. 2007.

[12] M. Abramowitz and I. E. Stegun, Handbook of mathematical functions with Formulas, Graphs and Mathematical Tables. New York: Dover Publications, Inc., 1970.

[13] A. M. Mathai and S. B. Provost, Quadratic Forms in Random Variables: Theory and Applications. Marcel Dekker, Inc, 1992.

[14] M. S. Alouini and A. J. Goldsmith, "Capacity of Rayleigh fading channels under different adaptive transmission and diversity combining techniques," IEEE Trans. Veh. Technol., vol. 48, no. 4, pp. 1165-1181, Jul. 1999.

[15] K. Cho and D. Yoon, "On the general BER expression of one- and twodimensional amplitude modulations," IEEE Trans. Commun., vol. 50, no. 7, pp. 1074-1080, Jul. 2002.

[16] G. T. Whyburn, "The Cauchy inequality in topological analysis," Proceedings of the National Academy of Sciences of the United States of America, vol. 48, no. 8, pp. 1335-1336, Aug. 1962. 